\title{
Minireview: Bacterial Sialyltransferases for Carbohydrate Synthesis
}

\author{
Oliver Schwardt\$^, Tamara Visekruna, Said Rabbani, and Beat Ernst \\ $\S$ SCS Poster Prize Winner
}

\begin{abstract}
Sialylation catalyzed by sialyltransferases is one of the most interesting enzymatic glycosyl transfer reactions, since chemical sialylations usually give only low yields and lead to poor stereoselectivities. In the last decade, several bacterial sialyltransferases were identified and found to exhibit broader substrate specificity than their mammalian counterparts. This suggests the potential usefulness of bacterial sialyltransferases in chemo-enzymatic synthesis of natural and non-natural sialooligosaccharides.
\end{abstract}

Keywords: Bacterial sialyltransferase $\cdot$ Chemo-enzymatic synthesis · Substrate specificity

\section{Introduction}

Sialic acid, often found in glycoproteins, glycolipids or polysaccharides, is associated with many important biological functions [1], which open up interesting therapeutic possibilities. In order to investigate these functions, and to develop novel carbohydrate-based therapeutics, libraries of diversified natural and modified sialooligosaccharides have to be synthesized and screened. Although recent progress in chemical sialylation reactions has facilitated the synthesis, it still remains one of the most difficult glycosylation reactions because of the hindered tertiary anomeric center, which often leads to unacceptably low yields, and the lack of a participating group to control the stereochemical outcome [2-5]. A convenient alternative is the

\footnotetext{
${ }^{\star}$ Correspondence: Dr. O. Schwardt University of Basel

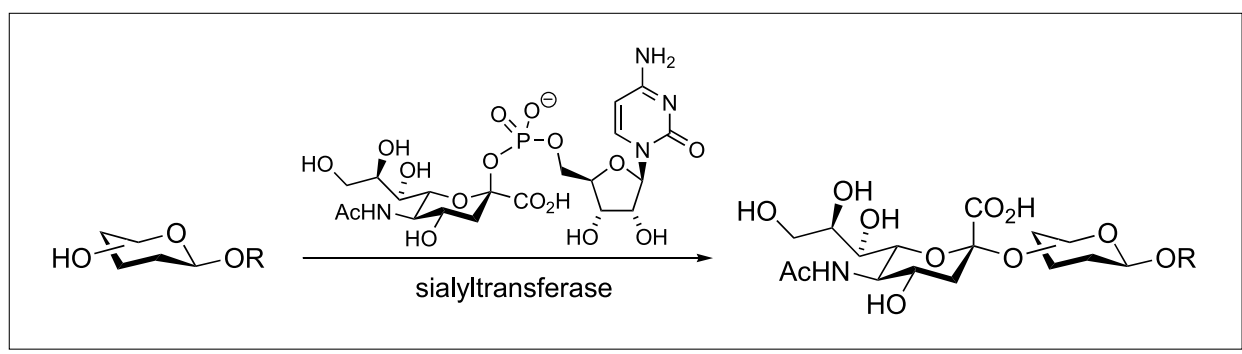

Scheme 1. Enzymatic sialylation of carbohydrates using sialyltransferases and CMP-NeuNAc

use of sialyltransferases (STs) (EC 2.4.99.-) [6][7], which catalyze the transfer of a sialic acid moiety from cytidine-5'-monophospho- $N$-acetylneuraminic acid (CMP-Neu$N A c) \alpha$-selectively to the 3 - or 6-position of a terminal galactose residue or to the hydroxyl group in the 8- or 9-position of a terminal sialic acid unit (Scheme 1).

The high substrate specificity of mammalian STs, however, limits their usefulness for the synthesis of oligosaccharide libraries. In addition, the expression of STs in mammalian expression systems is prohibitively expensive. Recently, initial representatives of bacterial STs have been identified [8-10]. They accomplish transformations analogous to mammalian enzymes, and are of high interest because they are available by inexpensive large-scale expression in bacterial systems. Furthermore, studies clearly revealed their broader substrate specificity compared to their mammalian counterparts [9-11]. Because of their extended substrate specificity, the EC-numbers of most bacterial STs have not yet been determined [12]

Most microbial strains expressing STs are pathogens like Neisseria gonorrhoeae,
Neisseria meningitides or Campylobacter jejuni. It has been shown that their surface lipooligosaccharides contain sialooligosaccharides identical to the structures found in mammalian glycolipids. This is presumably a form of molecular mimicry enabling the pathogens to evade the host's immune response [8][10].

In this minireview, we focus on the donor and acceptor specificity of bacterial STs and their application for the chemo-enzymatic synthesis of sialooligosaccharides.

\section{$\alpha(2 \rightarrow 3)$-Sialyltransferase from Neisseria gonorrhoeae}

The lst gene, encoding for $\alpha(2 \rightarrow 3)$ ST (EC 2.4.99.4) from Neisseria gonorrhoeae immunotype F62 (ATCC33084), was cloned and over-expressed in E. coli using a $C$-terminal $\mathrm{His}_{6}$-tag permitting an easy purification by Ni/NTA-affinity chromatography [13]. The acceptor specificity of the recombinant sialyltransferase was explored using synthetic oligosaccharides, sulfated oligosaccharides and glycolipids (Table 1) [13]. 
Table 1. Acceptor specificity of the recombinant $\alpha(2 \rightarrow 3)$-ST from $N$. gonorrhoeae F62 (EC 2.4.99.4) [13]

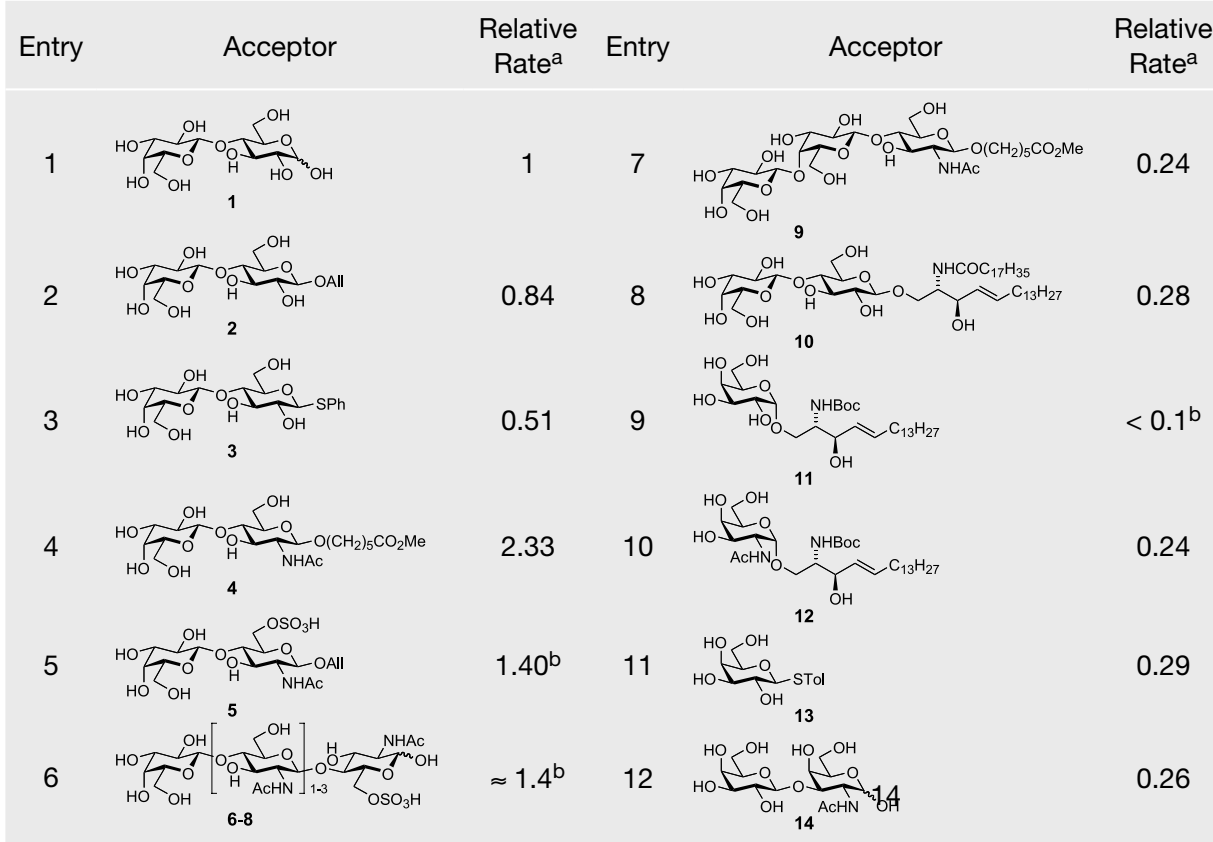

aBased on an isotope assay using CMP-[ $\left.{ }^{14} \mathrm{C}\right]-\mathrm{NeuNAc}$; b based on TLC and/or ESI-MS analysis.

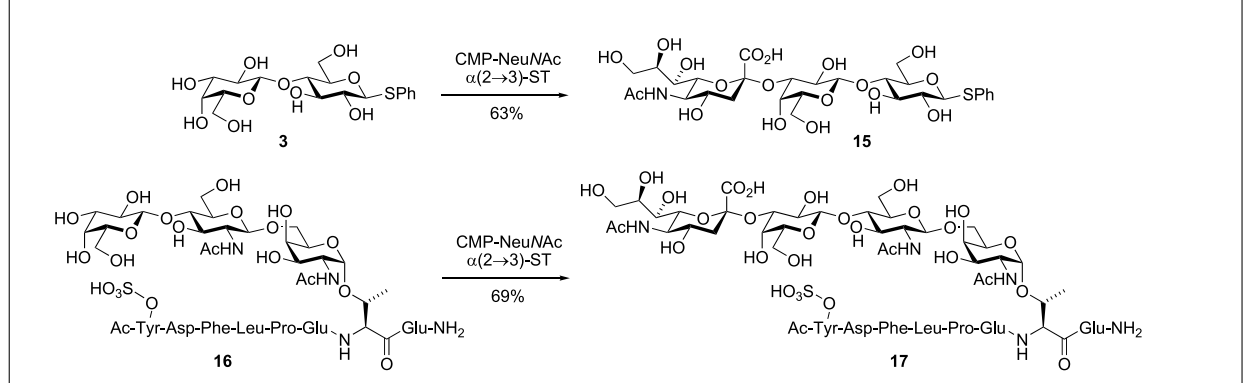

Scheme 2. Preparative sialylations with recombinant $N$. gonorrhoeae F62 $\alpha(2 \rightarrow 3)-S T$ (EC 2.4.99.4) [13][14]

Table 2. Acceptor specificity of the recombinant $N$. meningitides MC58(L3) $\alpha(2 \rightarrow 3)$-ST (EC 2.4.99.4) [15]

\begin{tabular}{|c|c|c|c|}
\hline Entry & Acceptor & Concentration [mM] & $\begin{array}{l}\text { Product } \\
\text { detected }\end{array}$ \\
\hline 1 & Gal $\alpha(1 \rightarrow 4)$ GlcNAc $\beta-O-A P-F C H A S E ~(18)$ & 0.5 & $++^{a}$ \\
\hline 2 & Gal $\alpha(1 \rightarrow 4)$ GlcNAc $\beta-O-T M R ~(19)$ & 0.5 & $+^{\mathrm{b}}$ \\
\hline 3 & Gal $\alpha(1 \rightarrow 3)$ GlcNAc $\beta-O-T M R(20)$ & 0.5 & $+^{\mathrm{b}}$ \\
\hline 4 & Gal $\alpha(1 \rightarrow 4)$ GlcNAc $\beta-S-A H-F C H A S E ~(21)$ & 0.8 & $+^{a}$ \\
\hline 5 & Gal $\alpha(1 \rightarrow 4)$ Glc $\beta$-O-AP-FCHASE (22) & 0.5 & $+^{\mathrm{a}}$ \\
\hline 6 & Gal $\alpha-O-A P-F C H A S E(23)$ & 0.5 & $+^{\mathrm{a}}$ \\
\hline 7 & Gal $\alpha$-S-AH-FCHASE (24) & 1.0 & $+^{a}$ \\
\hline 8 & Gal $\alpha-O-A P-F C H A S E(25)$ & 0.5 & $+^{a}$ \\
\hline 9 & Gal $\alpha(1 \rightarrow 4)$ Gal $\beta(1 \rightarrow 4)$ Glc $\beta$-O-AP-FCHASE (26) & 0.5 & $+^{a}$ \\
\hline 10 & Gal $\alpha(1 \rightarrow 4)[$ Fuc $\alpha 1 \rightarrow 3)]$ GlcNAc $\beta-O-T M R\left(\right.$ Le $\left.^{\mathrm{x}}, 27\right)$ & 0.5 & $-b$ \\
\hline 11 & Gal $\alpha(1 \rightarrow 3)[$ Fuc $\alpha(1 \rightarrow 4)]$ GlcNAc $\beta$-O-TMR $\left(\right.$ Le $\left.^{a}, 28\right)$ & 0.25 & $-b$ \\
\hline
\end{tabular}




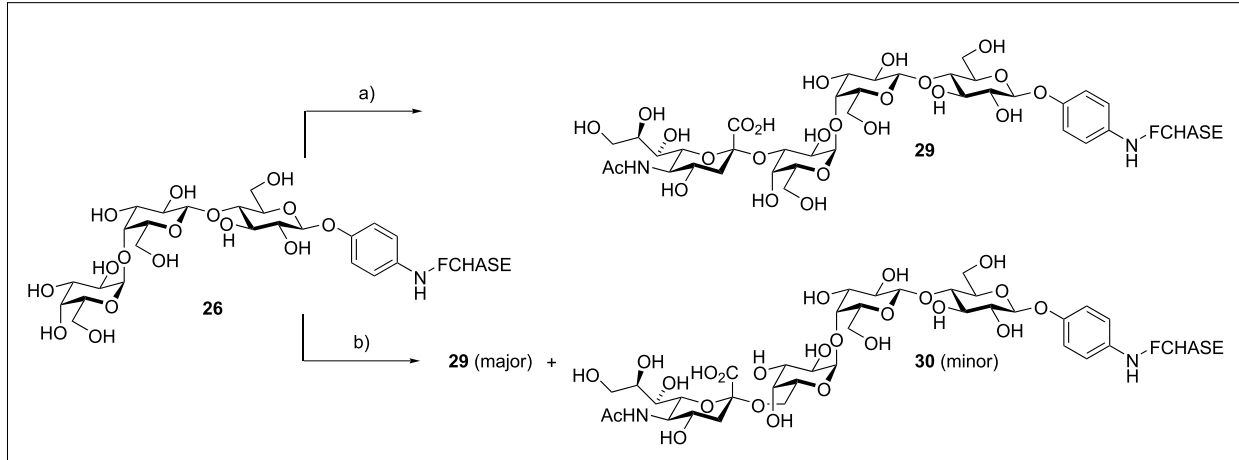

Scheme 3. Preparative sialylation of $\mathrm{P}^{\mathrm{k}}$-FCHASE 26 with CMP-NeuNAc and a) recombinant $\alpha(2 \rightarrow 3)$-ST from N. meningitides MC58(L3) [15]. b) bifunctional $\alpha(2 \rightarrow 3 / 6)-S T$ from $N$. meningitides 126E(L1) [16]

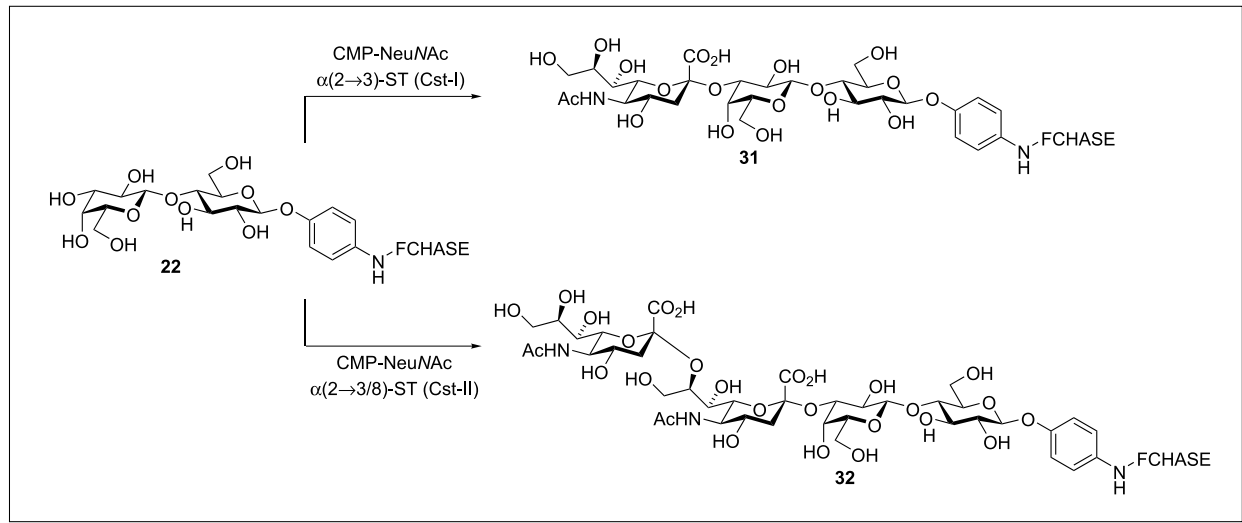

Scheme 4. Sialylation of Lac-FCHASE 22 with Cst-I and Cst-II from C. jejuni OH4384 [18]

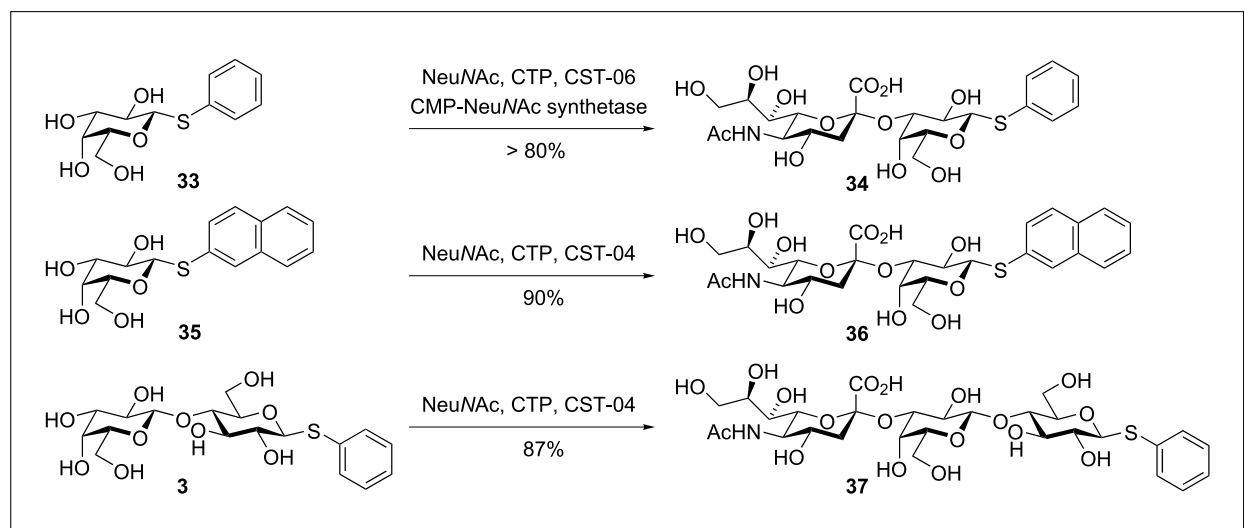

Scheme 5. Preparative syntheses of sialylated arylthio galactosides and lactoside with recombinant $\alpha(2 \rightarrow 3)$-ST (Cst-I) from C. jejuni OH4384 (EC 2.4.99.-) [19]

unit. However, both $\operatorname{Gal} \beta(1 \rightarrow 4) \mathrm{Glc} N A \mathrm{c}$ (compounds 18 and 19; entries $1 \& 2$ ) and $\mathrm{Gal} \beta(1 \rightarrow 3)$ GlcNAc (20, entry 3$)$ were accepted by the ST. The LacNAc-thioglycoside 21 (entry 4) and lactoside 22 (entry 5) were also sialylated. The enzyme tolerated the $\beta$-galactoside $\mathbf{2 3}$ as well as the Gal $\beta$ thioglycoside 24 (entries $6 \& 7$ ). In addition, the $\alpha$-galactosides $\mathbf{2 5}$ and $\mathbf{2 6}$ (entries $8 \& 9$ ) were found to be substrates. On the other hand, neither of the trisaccharides 27 $\left(\mathrm{Le}^{\mathrm{x}}\right)$ and $28\left(\mathrm{Le}^{\mathrm{a}}\right)$ were sialylated (entries 10 \& 11), exhibiting a behavior common to all known STs.

It was also shown that the activity of the recombinant $\alpha(2 \rightarrow 3)$-ST from $N$. meningit- ides MC58(L3) can be utilized for preparative sialylations $(\rightarrow \mathbf{2 9}$, Scheme 3a) [15].

In another study the same group reported on the ST activity from the cell-free extract of Neisseria meningitidis $126 \mathrm{E}(\mathrm{L} 1)$ [16]. The $126 \mathrm{E}(\mathrm{L} 1) \alpha(2 \rightarrow 3)-S T$ (Lst, EC 2.4.99.4) was assayed with $\mathrm{P}^{\mathrm{k}}$-FCHASE (26) as acceptor, and two products were detected by capillary electrophoresis. The major product was identified as the $\alpha(2 \rightarrow$ 3 )-sialylated product $\mathbf{2 9}$, whereas the minor fraction was found to be NeuNAca $(2 \rightarrow$ 6) $\mathrm{P}^{\mathrm{k}}$-FCHASE (30) (Scheme 3b). A sequence alignment of the two Lst's from $N$. meningitidis strains $126 \mathrm{E}(\mathrm{L} 1)$ and MC58(L3) revealed a difference in only six amino acids. By site-directed mutagenesis (Gly168Ile) in the MC58(L3)-Lst, the $\alpha(2 \rightarrow 6)-S T$ activity could be successfully improved, thus creating a new bifunctional $\alpha(2 \rightarrow 3 / 6)-S T$ (EC not determined).

One of the major drawbacks of preparative enzymatic sialylations is the cost and the lability of the donor CMP-NeuNAc. One possibility to overcome this handicap is the in situ formation of the sugar nucleotide from sialic acid and cytidine-5-triphosphate (CTP) by fusion enzymes with CMPNeuNAc synthetase and sialyltransferase activity. This methodology was successfully applied for the $100 \mathrm{~g}$-scale synthesis of 3'-sialyllactose from lactose using the recombinant fusion protein $\mathrm{CMP}-\mathrm{NeuNAc}$ synthetase/N. meningitidis MC58 (L3) $\alpha(2 \rightarrow 3)-$ ST [17].

\section{Sialyltransferases from Campylobacter jejuni}

Two ST genes were identified in the Campylobacter jejuni strain $\mathrm{OH} 4384$ by Wakarchuk and coworkers. [18]. These genes were designated as cst-I and cst-II, and then cloned and over-expressed in $E$. coli. The cst-I gene product transformed Lac-FCHASE 22 into NeuNAc $\alpha(2 \rightarrow$ 3)Lac-FCHASE 31 (Scheme 4), indicating that the Cst-I (EC 2.4.99.-) is an $\alpha(2 \rightarrow$ $3)-S T$. The cst-II gene product turned out to be a bifunctional $\alpha(2 \rightarrow 3 / 8)$-ST (EC 2.4.99.-) transforming Lac-FCHASE 22 into NeuNAc $\alpha(2 \rightarrow 8) \mathrm{NeuNAc} \alpha(2 \rightarrow 3) \mathrm{Lac}-$ FCHASE 32.

The recombinant Cst-I (EC 2.4.99.-) was used for the synthesis of thioacetals of 3-sialylgalactoside and 3'-sialyllactoside on a $100 \mathrm{mg}-1 \mathrm{~g}$-scale (Scheme 5) [19]. Sialylation of phenylthio $\beta$-galactoside (33) with CST-06, a fusion protein of Cst-I and maltose-binding protein (MBP), gave 3-sialylgalactoside $\mathbf{3 4}$ in $>80 \%$ yield. The MBP was used as an $N$-terminal tag allowing the easy purification of the enzyme by amylose affinity chromatography. Sialylation of 2naphtylthio $\beta$-galactoside (35) and phenylthio $\beta$-lactoside (3) with CST-04, a fusion protein of Cst-I and N. meningitidis CMPNeuNAc-synthetase, gave the corresponding sialosides $\mathbf{3 6}$ and $\mathbf{3 7}$ in $90 \%$ and $87 \%$ yield, respectively.

In 2004, the X-ray structure of $\Delta 32 \mathrm{Cst}$ II, a truncated form of the $C$. jejuni $\mathrm{OH} 4384$ Cst-II, co-crystallized with the inert donor substrate analog CMP-3-F-NeuNAc was published [20]. A careful analysis of the protein structure suggested a random point mutation (Ile53Ser) that would lead to the stabilization of the $\Delta 32 \mathrm{Cst}$-II construct by interacting with the acceptor substrates. It was further expected that the mutation would affect the relative ratios of $\alpha(2 \rightarrow$ 
Table 3. Relative acceptor substrate specificities of C. jejuni OH4384 Cst-II (EC 2.4.99.-) [21]

Entry Acceptor

\begin{tabular}{|c|c|c|}
\hline 1 & Gal $\beta(1 \rightarrow 4)$ Glc $(1)$ & \\
\hline 2 & Gal $\beta(1 \rightarrow 4) G l c \beta-R^{1}(38)$ & \\
\hline 3 & Gal $\beta(1 \rightarrow 4)$ GlcNAc (39) & \\
\hline 4 & Gal $\beta(1 \rightarrow 4) G I c N A c \beta-R^{1}(40)$ & \\
\hline 5 & Gal $\beta(1 \rightarrow 4)$ Glc $N A c \beta(1 \rightarrow 4)$ Gal $\beta(1 \rightarrow 4) G \mid c \beta-R^{1}(41)$ & \\
\hline 6 & Gal $\beta-R^{1}(42)$ & \\
\hline 7 & $\mathrm{Gal} \beta-\mathrm{SCH}\left(\mathrm{CH}_{3}\right)_{2}(\mathbf{4 3})$ & \\
\hline 8 & GalNAc $\beta-R^{1}(44)$ & \\
\hline
\end{tabular}

$9 \quad$ NeuNAc $\alpha(2 \rightarrow 3)$ Gal $\beta(1 \rightarrow 4) G l c \beta-R^{2}(45)$

100

63

199

116

43

12

14 NeuNAc $\alpha(2 \rightarrow 8) \operatorname{NeuNAc} \alpha(2 \rightarrow 3) \operatorname{Gal} \beta(1 \rightarrow 4) \operatorname{Glc} \beta-R^{2}(\mathbf{5 0}, \mathrm{GD} 3)$

15 NeuNAc $\alpha(2 \rightarrow 8) \operatorname{NeuNAc\alpha }(2 \rightarrow 8) \operatorname{NeuNAc\alpha }(2 \rightarrow 3) \operatorname{Gal} \beta(1 \rightarrow 4) G \mid c \beta-R^{2}(\mathbf{5 1}$, GT3)

$16 \quad \operatorname{NeuNGc\alpha }(2 \rightarrow 3) \operatorname{Gal} \beta(1 \rightarrow 4) \operatorname{Glc} N A c \beta-R^{2}(52)$

aRelative specific activity for each compound is calculated as a percentage of the reference compound (bold).

$\mathrm{R}^{1}=\mathrm{O}\left(\mathrm{CH}_{2}\right)_{2} \mathrm{~N}_{3} ; \mathrm{R}^{2}=\mathrm{O}\left(\mathrm{CH}_{2}\right)_{2} \mathrm{NHFmoc}$.

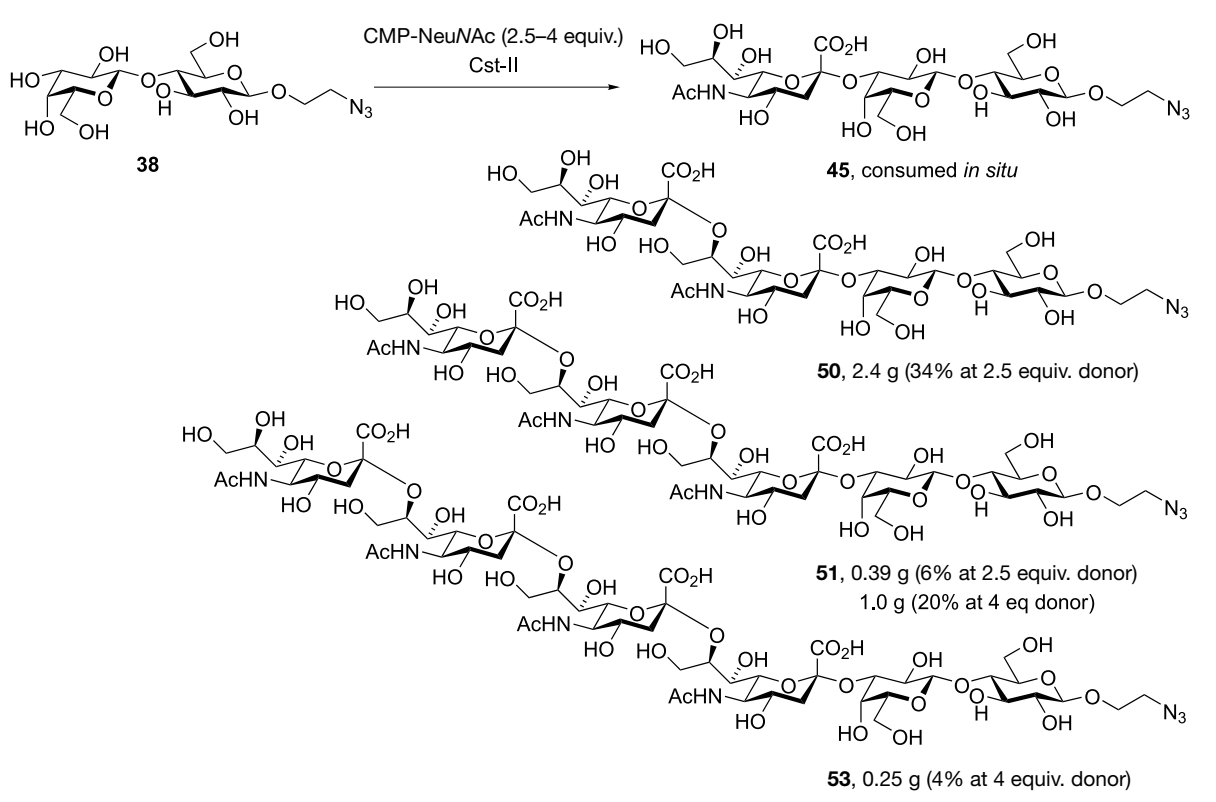

Scheme 6. Preparative synthesis of GD3 (50), GT3 (51) and poly-sialylated oligosaccharides with recombinant $\alpha(2 \rightarrow 3 / 8)$-ST (Cst-II) from C. jejuni OH4384 (EC 2.4.99.-) [21]

3)- and $\alpha(2 \rightarrow 8)$-activities and enhance the $\alpha(2 \rightarrow 8)$-specificity.

Recently, the relative $\alpha(2 \rightarrow 3)$ - and $\alpha(2 \rightarrow 8)$-activities of the mutated Cst-II (Ile53Ser) were evaluated (Table 3) [21]. To minimize $\alpha(2 \rightarrow 8)$-sialylation, the Cst-II $\alpha(2 \rightarrow 3)$-activity was measured using high substrate concentrations and short incubation times.

Lactose (1) and lactoside 38 (entries 1 $\& 2)$ had the highest $\alpha(2 \rightarrow 3)$-activity of all compounds tested. $N$-Acetyllactosamines (compounds 39 and 40, entries $3 \& 4$ ) also demonstrated excellent activity, which is still sufficient for preparative applications. Extended LacNAc 41 (entry 5), however, showed a substantially reduced activity. Interestingly, the capability of $\beta$-galactosides 42-44 (entries 6-8) to act as acceptors was influenced dramatically by the reducing end substituent. Although $\mathbf{4 2}$ could be easily transformed into the corresponding sialoside, isopropylthio $\beta$-galactoside (43) and GalNAc 44 were not tolerated by the enzyme.

The $\alpha(2 \rightarrow 8)$-activity study showed that both $\alpha(2 \rightarrow 3)$-sialosides (45-47, entries 9$11)$ and $\alpha(2 \rightarrow 6)$-sialosides $(\mathbf{4 7}, \mathbf{4 8}$; entries $12 \& 13$ ) were excellent substrates for CstII. The larger oligosaccharides GD3 (50) and GT3 (51) (entries 14 \& 15) showed significantly reduced acceptor qualities. It is noteworthy, however, that the $C$. jejuni $\mathrm{OH} 4384$ Cst-II is also able to synthesize polysialosides. In addition, NeuNGco $(2 \rightarrow$ 3)LacNAc (52) (entry 16), containing a glycolated sialic acid, turned out to be an excellent substrate.

Finally, the $C$. jejuni OH4384 Cst-II was also used for the preparative synthesis on a gram-scale of ganglioside derivatives starting from lactoside 38 (Scheme 6). The originally formed 3'-sialyllactoside $\mathbf{4 5}$ was completely consumed and converted to GD3 (50) and GT3 (51). Even a small amount of tetrasialyl-lactoside $\mathbf{5 3}$ could be isolated. In addition, traces of higher sialylated fractions were also detected but not purified. With increasing amounts of CMP-NeuNAc, the formation of $\alpha(2 \rightarrow 8)$-polysialosides could be significantly raised [21].

Recently, we over-expressed the truncated cst-II gene from $C$. jejuni strain ATCC43438 encoding for $\Delta 32$ Cst-II (EC not determined) in $E$. coli, again using a $C$-terminal $\mathrm{His}_{6}$-tag for affinity chromatography purification [22]. The $\alpha(2 \rightarrow 3)$ - and $\alpha(2 \rightarrow 8)$-specificity of the enzyme was assayed using the type I and II disaccharides $\mathbf{5 4}$ and 56, as well as the corresponding mono-sialylated trisaccharides $\mathbf{5 5}$ and $\mathbf{5 7}$ (Table 4). All reactions were run on a preparative scale and the products analyzed by ${ }^{1} \mathrm{H}$ and ${ }^{13} \mathrm{C}$ NMR.

The $\alpha(2 \rightarrow 3)$-activity of the wild-type $\Delta 32$ Cst-II was significantly higher for the type I disaccharide 54 (entry 1) than for LacNAc (56, entry 3). On the other hand, no $\alpha(2 \rightarrow 8)$-activity was observed with the type I sugars 54 and 55 (entries 1 \& 2). The type II substrates $\mathbf{5 6}$ and $\mathbf{5 7}$ (entries 3 \& 4), however, were partly converted into the corresponding bis-sialylated tetrasaccharide NeuNAco $(2 \rightarrow 8)$ NeuNAco $\alpha(2 \rightarrow$ $3) \mathrm{Gal} \beta(1 \rightarrow 4) \mathrm{Glc} N A c \beta-O H e x$, but with a much lower rate as described for the $C$. $j e$ juni OH4384 Cst-II [21].

The sequences of the Cst-IIs from C. jejuni strains OH4384 and ATCC43438 differ by only six amino acids ( $97.3 \%$ sequence identity). The analysis of the $\mathrm{X}$-ray struc- 
ture of the OH4384 $\Delta 32 \mathrm{Cst}$-II [20] revealed two neutral amino acids, i.e. Asn177 and Asn182 close to the binding site, whereas the same positions in the ATCC43438 CstII enzyme are occupied by a negatively charged Asp177 and a positively charged Arg182. Based on molecular modeling studies, it was assumed that replacement of these charged residues might increase the $\alpha(2 \rightarrow 8)$-activity of the enzyme. Therefore, the mutant $\Delta 32$ Cst-II (S53N177N182) was generated by site-directed mutagenesis (Gly53Ser, Asp177Asn, Arg182Asn), and the specificity of this enzyme evaluated again with carbohydrates 54-57 [22].

The mutant $\Delta 32$ Cst-II (S53N177N182) acted as a bifunctional $\alpha(2 \rightarrow 3 / 8)$-sialyltransferase with both types of acceptors (Table 4). In the case of the type I disaccharide 54 (entry 1$)$, the $\alpha(2 \rightarrow 3)$-activity remained unchanged compared to the wild-type enzyme, but the mutant showed additional $\alpha(2 \rightarrow 8)$ activity. Surprisingly, no significant change of either the $\alpha(2 \rightarrow 3)$ - or the $\alpha(2 \rightarrow 8)$-activity was found for the type II substrate 56 (entry 3). In addition, the mutant accepted both trisaccharides $\mathbf{5 5}$ and $\mathbf{5 7}$ as substrates with a slightly higher activity for the type II acceptor 57 (entry 4). The conversion rates for both substrates did not exceed 50\%. Running the reactions with higher amounts of CMP-Neu$N A c$ as well as adding more donor or transferase during the incubation did not influence this outcome. In contrast to findings by Blixt et al. [21] with the $C$. jejuni $\mathrm{OH} 4384$ Cst-II, formation of multi-sialylated products could not be observed.

\section{$\alpha(2 \rightarrow 3)$-Sialyltransferase from Haemophilus influenzae}

The ST gene of the human pathogen Haemophilus influenzae, responsible for linking NeuNAc in an $\alpha(2 \rightarrow 3)$-manner to lactose, was identified in 2001 [23]. It was cloned and over-expressed in E. coli and the $\alpha(2 \rightarrow 3)$-ST (EC not determined) activity determined by TLC or capillary electrophoresis using Lac-FCHASE as substrate. The acceptor specificity of the enzyme was examined using FCHASE-labeled acceptors, and ranked in the following manner: LacFCHASE (22) = LacNAc-FCHASE (18) > Gal $\alpha$-FCHASE (25) $>$ Gal $\beta$-FCHASE (23). $\mathrm{P}^{\mathrm{k}}$-FCHASE (26) was not tolerated by the enzyme [23].

\section{$\alpha(2 \rightarrow 6)$-Sialyltransferase from Photobacterium damsela}

The purification of an ST from the marine bacterium Photobacterium damsela JT0160 was reported by a group from Japan Tobacco Inc. [9]. The enzyme was found to exhibit $\alpha(2 \rightarrow 6)$-activity. In a screening

Table 4. Sialylation of type I and II saccharides with recombinant $\alpha(2 \rightarrow 3 / 8)$-ST ( $(32$ Cst-II) from $C$. jejuni ATCC43438 [22]

Entry

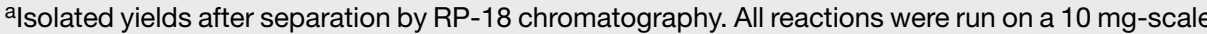
with 2.5 equiv. of donor CMP-NeuNAc for disaccharides and 1.5 equiv. for trisaccharides. Hex = $n$-hexyl.

Table 5. Transfer assay of the P. damsela $\alpha(2 \rightarrow 6)$-ST (EC 2.4.99.1) with PA-labeled oligosaccharides [24]

\begin{tabular}{|c|c|c|}
\hline Entry & Acceptor & $\begin{array}{c}\text { Transfe } \\
\text { yielda }^{\mathrm{a}} \\
{[\%]}\end{array}$ \\
\hline 1 & NeuNAc $\alpha(2 \rightarrow 3)$ Gal $\beta(1 \rightarrow 4)$ Glc-PA (58) & 28 \\
\hline 2 & NeuNGc $\alpha(2 \rightarrow 3)$ Gal $\beta(1 \rightarrow 4)$ Glc-PA (59) & 78 \\
\hline 3 & NeuNAc $\alpha(2 \rightarrow 8)$ NeuNAc $\alpha(2 \rightarrow 3)$ Gal $\beta(1 \rightarrow 4)$ Glc-PA $(60)$ & 76 \\
\hline 4 & Fuc $\alpha(1 \rightarrow 2)$ Gal $\beta(1 \rightarrow 4)$ Glc-PA $(61)$ & 57 \\
\hline 5 & Gal $\beta(1 \rightarrow 4)$ Glc $N A c \beta(1 \rightarrow 3)$ Gal $\beta(1 \rightarrow 4)$ Glc-PA $(62)$ & 44 \\
\hline 6 & Gal $\beta(1 \rightarrow 4)[$ Fuc $\alpha(1 \rightarrow 3)]$ Glc $N A c \beta(1 \rightarrow 3)$ Gal $\beta(1 \rightarrow 4)$ Glc-PA $\left(63\right.$, Le $\left.^{x}\right)$ & $<5$ \\
\hline 7 & Gal $\beta(1 \rightarrow 3)[$ Fuc $\alpha(1 \rightarrow 4)]$ Glc $N A c \beta(1 \rightarrow 3)$ Gal $\beta(1 \rightarrow 4)$ Glc-PA $\left(64\right.$, Le $\left.^{a}\right)$ & $<5$ \\
\hline 8 & Gal $\beta(1 \rightarrow 3)$ Gal $N A c \beta(1 \rightarrow 4)[$ Neu $N A c \alpha(2 \rightarrow 3)]$ Gal $\beta(1 \rightarrow 4)$ Glc-PA $(65)$ & $<5$ \\
\hline 9 & $\begin{array}{l}\text { NeuNAc } \alpha(2 \rightarrow 3) \text { Gal } \beta(1 \rightarrow 3) \text { GalNAc } \beta(1 \rightarrow 4)[\operatorname{NeuNAc} \alpha(2 \rightarrow 3)] \text { Gal } \beta(1 \rightarrow 4) \text { Glc-PA } \\
(66)\end{array}$ & $<5$ \\
\hline 10 & $\begin{array}{l}\text { Gal } \beta(1 \rightarrow 3) \text { Gal } N A c \beta(1 \rightarrow 4)[\operatorname{NeuNAc} \alpha(2 \rightarrow 8) \operatorname{NeuNAc} \alpha(2 \rightarrow 3)] \text { Gal } \beta(1 \rightarrow 4) \text { Glc-PA } \\
(67)\end{array}$ & $<5$ \\
\hline
\end{tabular}

aYields were estimated by HPLC. PA = 2-pyridylamino

study with various mono- and disaccharides using ${ }^{14} \mathrm{C}$-labeled CMP-NeuNAc as donor, Gal $\alpha / \beta-O M e$, GalNAc $\alpha-O M e$, LacNAc $\beta$ $\mathrm{OMe}$ and lactose were found to be good substrates for the $P$. damsela JT0160 $\alpha(2 \rightarrow$ 6)-ST (Bst, EC 2.4.99.1). The $K_{m}$ value for lactose was almost the same as for LacNAc, whereas that of methyl $\beta$-galactoside was about 25 times higher.
A transfer assay with the $P$. damsela JT0160 $\alpha(2 \rightarrow 6)$-ST using 2-pyridylamino (PA)-labeled oligosaccharides revealed a remarkable ability of this enzyme to sialylate the 6-position of terminal, but also mono- and bis-sialylated galactose moieties (Table 5) [24].

Whereas 3'-sialyllactoside 58 (entry 1) was a moderate substrate, its $N$-glycolyl- 
neuraminyl-analogue 59 (entry 2) and the bis-sialylated lactoside $\mathbf{6 0}$ (entry 3 ) gave excellent transfer results. 2'-Fucosyllactoside 61 (entry 4) and extended LacNAc 62 (entry $5)$ were also accepted by the enzyme. The $K_{m}$ values of 3'-sialyllactoside 58 and 2'-fucosyllactoside $\mathbf{6 1}$ were found to be similar to those of lactose and $\mathrm{N}$-acetyllactosamine [24]. However, Le ${ }^{\mathrm{x}}$ (63, entry 6), Le (64, $^{\mathrm{a}}$ entry 7) and the oligosaccharides 65-67 (entries 8-10), which contain $\beta(1 \rightarrow 3)$-linked galactose, were not sialylated.

For the application of the enzyme in preparative synthesis, a large-scale production of the $\alpha(2 \rightarrow 6)-S T$ was established by cultivating $P$. damsela JT0160 cells in culture media containing galactose and beef extract at $\mathrm{pH}$ 8. The crude enzyme extract obtained in the supernatant proved to be sufficiently pure for synthetic applications [25a]. The ST gene (bst) was also cloned and over-expressed in E. coli MV1184, and the recombinant gene product gave a soluble form of the enzyme [25b].

The following oligosaccharides were synthesized in 50-90\% yield and fully characterized by ${ }^{1} \mathrm{H}$ and ${ }^{13} \mathrm{C}$ NMR (Scheme 7) [25]: 3',6'-Disialyllactose (68) and 2'fucosyl-6'-sialyllactose (69) were synthesized on a 6-8 mg scale. 6-Sialylated lactose (70), methyl $\beta$-galactoside (71) and $\mathrm{N}$-acetylgalactosamine (72) were obtained on a $c a .5 \mathrm{mg}$ scale using the crude enzyme extract. 6'-KDN-lactose (73) was also synthesized using CMP-KDN as donor.

Recently, the $\alpha(2 \rightarrow 6)$-ST from P. damsela was also applied for the enzymatic sialylation of GalNAc, $\alpha$-linked either to a serine or threonine residue of the $\mathrm{T}_{\mathrm{N}}$-glycopeptides 74 and 75 (Scheme 8) [26]. In contrast to the mammalian sialyltransferase ST6GalNAc I (EC 2.4.99.3), which sialylates only the GalNAc $\alpha$-Thr moiety to

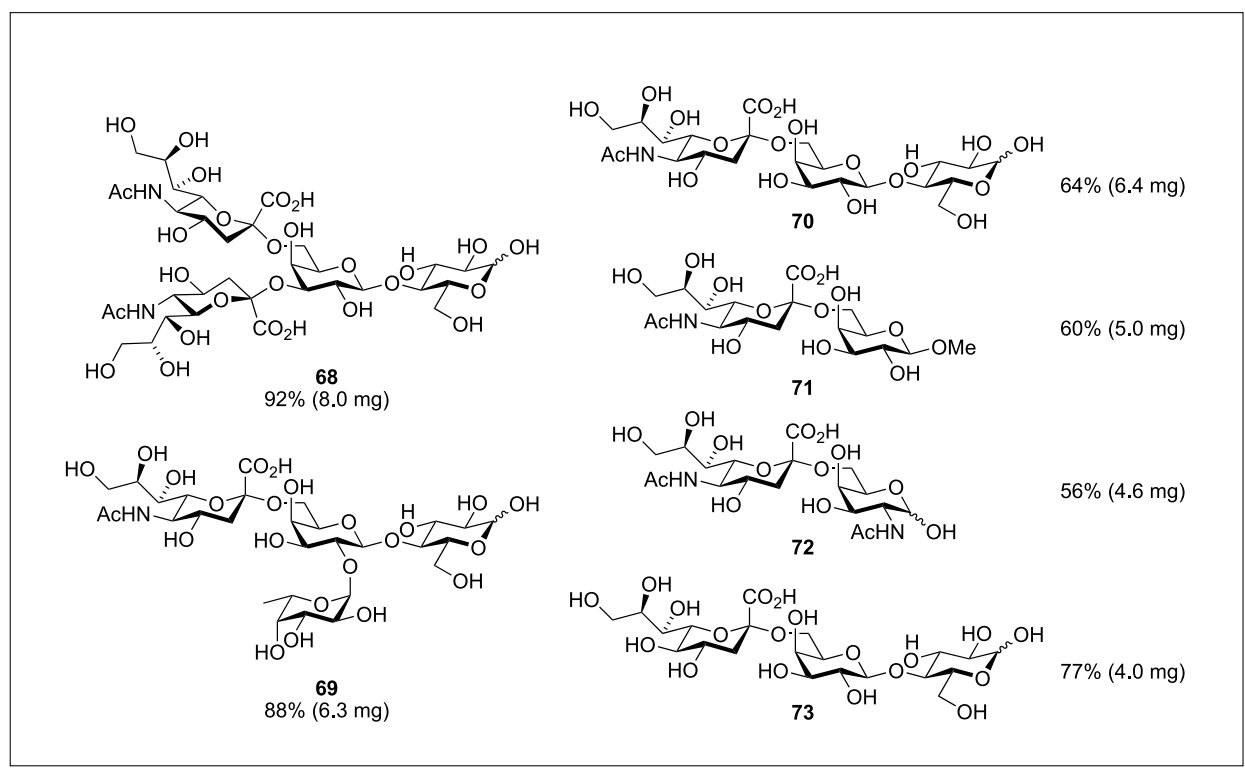

Scheme 7. Natural and non-natural sialooligosaccharides synthesized with $P$. damsela $\alpha(2 \rightarrow 6)-\mathrm{ST}$ (EC 2.4.99.1) [25]

give 76, the bacterial enzyme also accepts GalNAc $\alpha$-Ser as a substrate $(\rightarrow 77)$.

\section{Synthesis of Sialooligosaccharides Using Metabolically Engineered Whole Cells}

Sialooligosaccharides and CMP-NeuNAc were also synthesized on a large scale using metabolically engineered whole cells as reactors. For the production of CMPNeuNAc, two recombinant $E$. coli strains (E. coli $\mathrm{K} 1$ ), over-expressing CMP-NeuNAc synthetase (EC 2.7.7.43) and CTP synthetase (EC 6.3.4.2), were generated [27] and combined in a reaction vessel with Corynebacterium ammoniagenes, which is responsible for the production of UTP from orotic acid. In this system, con- sisting of three cell lines, CMP-NeuNAc was accumulated after $1 \mathrm{~d}$ of incubation up to a concentration of $27 \mathrm{mM}(17 \mathrm{~g} / \mathrm{l})$. When another E. coli strain, over-expressing the $N$. gonorrhoeae $\alpha(2 \rightarrow 3)$-ST gene, was added to this system, 3 '-sialyllactose was produced up to a maximal concentration of $52 \mathrm{mM}(33 \mathrm{~g} / \mathrm{l})$ after $11 \mathrm{~h}$ of reaction. Incubation on different scales $(30 \mathrm{ml}$ and 2 1) gave comparable results (Scheme 9) [27].

NeuNAc $\alpha(2 \rightarrow 3)$ GalNAc was also obtained on a $1 \mathrm{~g}$-scale by coupling two bacterial expression systems (the CMPNeuNAc production system and an $E$. coli strain over-expressing $P$. damsela $\alpha(2 \rightarrow$ 6)-ST) [28].

Recently, the oligosaccharide moieties of the gangliosides GM3 (3'-sialyllactose) and GD3 [NeuNAc $\alpha(2 \rightarrow 8) \operatorname{NeuNAc} \alpha(2 \rightarrow$

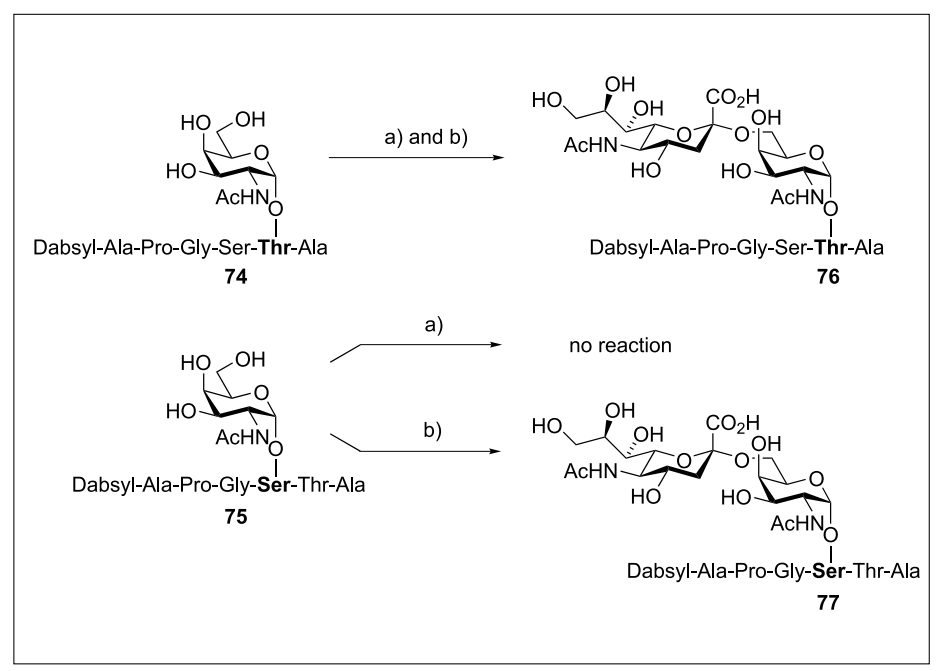

Scheme 8. Preparative sialylations of $\mathrm{T}_{\mathrm{N}}$-glycopeptides 74 and 75 [26] with CMP-NeuNAc and a) mammalian ST6GalNAc I (EC 2.4.99.3). b) P. damsela $\alpha(2 \rightarrow 6)$-ST (EC 2.4.99.1). Dabsyl = 4-dimethylaminoazobenzene-4'-sulfonyl

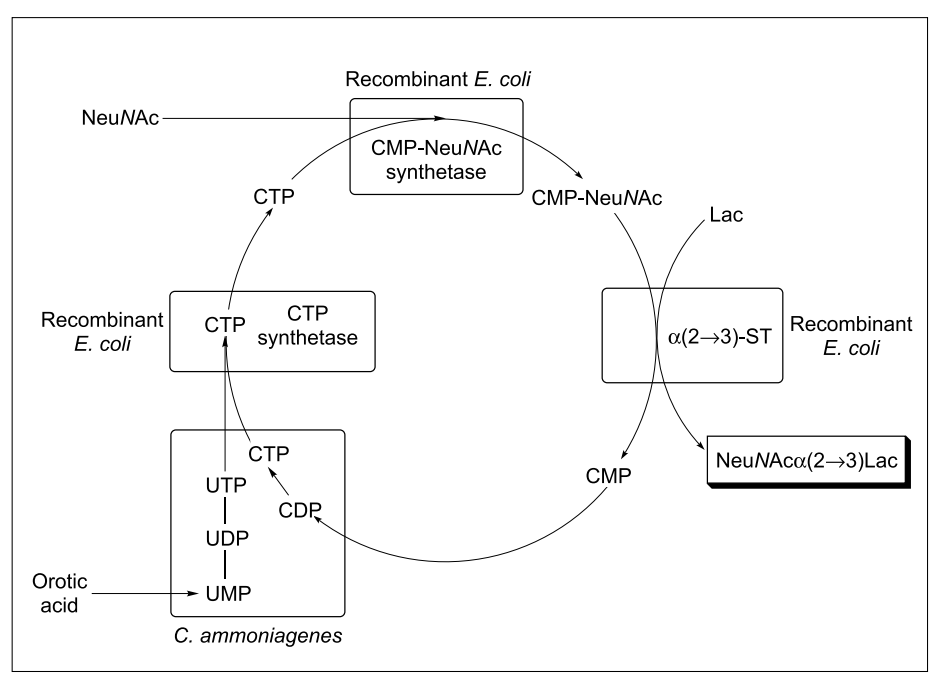

Scheme 9. Bacterial system consisting of four components for the synthesis of 3'-sialyllactose [27] 
3) Gal $\beta(1 \rightarrow 4)$ Glc] were synthesized by incubating lactose and sialic acid for $9 \mathrm{~h}$ with a metabolically engineered $E$. coli strain (E. coli K12) over-expressing $N$. meningitides CMP-NeuNAc synthetase (EC 2.7.7.43) and the bifunctional C. jejuni ATCC43438 $\alpha(2 \rightarrow 3 / 8)-S T$ (Cst-II) [29]. From 11 of culture $49 \mathrm{mg}$ of GM3 and $98 \mathrm{mg}$ of GD3 could be isolated. By a prolongation of the incubation time to $24 \mathrm{~h}, \mathrm{GD} 3$ was partially further sialylated to yield $15 \mathrm{mg}$ of tri-sialylated product GT3 [NeuNAc $\alpha(2 \rightarrow 8) \mathrm{NeuNAc} \alpha(2 \rightarrow$ $8) \mathrm{Neu} N \mathrm{Ac} \alpha(2 \rightarrow 3) \mathrm{Gal} \beta(1 \rightarrow 4) \mathrm{Glc}]$.

\section{Future Perspectives}

Enzymatic sialylations with sialyltransferases have substantially improved the glycosylation repertoire. They are superior to chemical sialylation in terms of stereoselectivity and overall yield. Additional advantages are reduced cost and labor, since there is no need for multiple protection-deprotection steps and cumbersome separation of isomers. Drawbacks of the enzymatic approach, however, are the difficulty of over-expression of mammalian enzymes and the prohibitively expensive sugar nucleotide CMP-NeuNAc. Since mammalian STs usually exhibit high substrate specificities, their use is often limited to the synthesis of natural products.

The herein-reviewed STs from bacterial sources, however, frequently show broader substrate specificity for both acceptors and donors, suggesting their usefulness not only in the chemo-enzymatic synthesis of natural, but also non-natural sialooligosaccharides and glycoconjugates, such as sulfated oligosaccharides or thioglycosides. Initial examples clearly demonstrated that the substrate tolerance can be further broadened by simple point mutations, offering the unique chance to engineer STs with tailor-made specificities. In addition, the large-scale production of catalysts in bacterial expression systems is considerably less expensive than the expression in mammalian or insect cells.

A new era in enzymatic sialylation became possible with the fusion protein technology and the utilization of whole cells as production systems of sialoglycans. Finally, the search for additional bacterial enzymes may provide new catalysts with surprising substrate specificities.

Received: February 19, 2006

[1] a) J.C. McAucliffe, O. Hindsgaul, Chem. Ind. 1997, 3, 170; b) R.A. Dwek, Chem. Rev. 1996, 96, 683; c) A. Varki, Glycobiology 1992, 2, 25.

[2] G.-J. Boons, A.-V. Demochemko, Chem. Rev. 2000, 100, 4539.
[3] a) J.C. Castro-Palomino, Y.E. Tsvetkov, R. Schneider, R.R. Schmidt, Tetrahedron Lett. 1997, 38, 6837; b) O. Kanie, O. Hindsgaul, Curr. Opin. Struct. Biol. 1992, 2, 674; c) M.P. DeNinno, Synthesis 1991, 583; d) K. Okamoto, T. Goto, $T e-$ trahedron 1990, 46, 5835.

[4] a) T. Ercégovic, G. Magnusson, J. Org. Chem. 1995, 60, 3378; b) T. Ercégovic, G. Magnusson, J. Org. Chem. 1996, 61, 179 ; c) N. Hossain, G. Magnusson, Tetrahedron Lett. 1999, 40, 2217.

[5] a) Z. Wang, X.F. Zhang, Y. Ito, Y. Nakahara, T. Ogawa, Bioorg. Med. Chem. 1996, 4, 1901; b) Y. Ito, S. Nunomura S. Shibayama, T. Ogawa, J. Org. Chem. 1992, 57, 1821.

[6] a) K.M. Koeller, C.-H. Wong, Chem. Rev. 2000, 100, 4465; b) X. Qian, K. Sujino, M.M. Palcic, in 'Carbohydrates in Chemistry and Biology', Eds. B. Ernst, G.W Hart, P. Sinaÿ, Wiley-VCH, Weinheim, 2000, Vol. 2, pp 685; c) S.L. Flitsch, Curr. Opin. Chem. Biol. 2000, 4, 619; d) Z. Guo, P.G. Wang, Appl. Biochem. Biotech. 1997, 68, 1; e) Y. Ichikawa, in 'Glycopeptides and related compounds', Eds. D.G. Large, C.D. Warren, Marcel Dekker, New York, 1997, pp. 29.

[7] a) R. Öhrlein, Topics Curr. Chem. 1999, 200, 227; b) B. Ernst, R. Oehrlein, Glycoconj. J. 1999, 16, 161; c) R. Öhrlein, Mini Rev. Med. Chem. 2001, 1, 349.

[8] a) H. Smith, N.J. Parsons, J.A. Cole, $M i-$ crob. Pathog. 1995, 19, 365; b) R. Rest, R.E. Mandrell, Microb. Pathog. 1995 19, 379; c) G.O. Aspinalli, A.G. McDonald, H. Pang, L.A. Kurjanczyk, J.L. Penner, Biochemistry 1994, 33, 241; d) R.E. Mandrell, J.M. Griffiss, H. Smith, J.A. Cole, Microb. Pathog. 1993, 14, 315.

[9] T. Yamamoto, M. Nakashizuka, H. Kodama, Y. Kajihara, I. Terada, J. Biochem. 1996, 120, 104.

[10] M. Gilbert, D.C. Watson, A.M. Cunningham, M.P. Jennings, N.M. Young, W.W. Wakarchuk, J. Biol. Chem. 1996, 271, 28271

[11] M. Izumi, C.-H. Wong, Trends Glycosci. Glycotech. 2001, 13, 345.

[12] P.M. Coutinho, B. Henrissat, Carbohydrate-Active Enzymes Server, 1999, http://afmb.cnrs-mrs.fr/CAZY/

[13] M. Izumi, G.-J. Shen, S. WacowichSgarbi, T. Nakatani, O. Plettenburg, C.H. Wong, J. Am. Chem. Soc. 2001, 123, 10909 .

[14] K.M. Koeller, M.E.B. Smith, R.-F. Huang, C.-H. Wong, J. Am. Chem. Soc. 2000, 122, 4241.

[15] M. Gilbert, A.M. Cunningham, D.C. Watson, A. Martin, J.C. Richards, W.W. Wakarchuk, Eur. J. Biochem. 1997, 249, 187.

[16] W.W. Wakarchuk, D. Watson, F. St. Michael, J. Li, Y. Wu, J.R. Brisson, N.M. Young, M. Gilbert, J. Biol. Chem. 2001, 276,12785 .
[17] M. Gilbert, R. Bayer, A.M. Cunningham, S. DeFrees, Y. Gao, D.C. Watson, N.M. Young, W.W. Wakarchuk, Nat. Biotechnol. 1998, 16, 769.

[18] M. Gilbert, J.R. Brisson, M.F. Karwaski, J. Michniewicz, A.M. Cunningham, Y. Wu, N.M. Young, W.W. Wakarchuk, J. Biol. Chem. 2000, 275, 3896.

[19] S. Mehta, M. Gilbert, W.W. Wakarchuk, D.M. Whitfield, Org. Lett. 2000, $2,751$.

[20] C.P.C. Chiu, A.G. Watts, L.L. Lairson, M. Gilbert, D. Lim, W.W. Wakarchuk, S.G. Withers, N.C.J. Strynadka, Nat. Struct. Mol. Biol. 2004, 11, 163.

[21] O. Blixt, D. Vasiliu, K. Allin, N. Jacobsen, D. Warnock, N. Razi, J.C. Paulson, S. Bernatchez, M. Gilbert, W. Wakarchuk, Carbohydr. Res. 2005, 340, 1963.

[22] O. Schwardt, T. Visekruna, G. Zenhäusern, S. Rabbani, B. Ernst, submitted.

[23] D.W. Hood, A.D. Cox, M. Gilbert, K. Makepeace, S. Walsh, M.E. Deadman, A. Cody, A. Martin, M. Mansson, E.K. Schweda, J.R. Brisson, J.C. Richards, E.R. Moxon, W.W. Wakarchuk, Mol. Microbiol. 2001, 39, 341.

[24] Y. Kajihara, T. Yamamoto, H. Nagae, M. Nakashizuka, T. Sakakibara, I. Terada, $J$. Org. Chem. 1996, 61, 8632.

[25] a) T. Yamamoto, H. Nagae, Y. Kajihara, I. Terada, Biosci. Biotechnol. Biochem. 1998, 62, 210; b) T. Yamamoto, M. Nakashizuka, I. Terada, J. Biochem. 1998, 123, 94.

[26] C.-F. Teo, T.-S. Hwang, P.-H. Chen, C.-H. Hung, H.-S. Gao, L.-S. Chang, C.-H. Lin, Adv. Synth. Catal. 2005, 347, 967.

[27] T. Endo, S. Koizumi, K. Tabata, A. Ozaki, Appl. Microbiol. Biotechnol. 2000, 53, 257.

[28] T. Endo, S. Koizumi, K. Tabata, S. Kakita, A. Ozaki, Carbohydr. Res. 2001, 330, 439.

[29] T. Antoine, A. Heyraud, C. Bosso, E. Samain, Angew. Chem. Int. Ed. 2005, 44, 1350 . 